\title{
APLICACIÓN DE ALGORITMOS GENÉTICOS SPEA \& MAGNO PARA DISEÑO ÓPTIMO DE REDES DE DISTRIBUCIÓN DE AGUA, VIÑANI, TACNA
}

\author{
APPLICATION OF SPEA \& MAGNO GENETIC ALGORITHMS FOR \\ OPTIMAL DESIGN OF NETWORKS WATER DISTRIBUTION, VIÑANI, \\ TACNA
}

\author{
${ }^{1}$ Edwin Pino Vargas, ${ }^{2}$ Violeta Quevedo Porras, ${ }^{3}$ Luis Alfaro Ravello, ${ }^{4}$ César Avendaño Jihuallaga
}

\begin{abstract}
RESUMEN
El diseño óptimo de redes de distribución es consecuencia de un estudio hidráulico detallado; el cual se ha adaptado a los criterios de ingeniería, a las exigencias económicas y a la normativa vigente. Yates, Templeman, \& Boffey (1984) afirman que se necesita encontrar un método aproximado efectivo que garantice calidad en los diseños para extender la cobertura del servicio de agua potable. En este contexto, se aplica el Algoritmo Evolutivo Multiobjetivo (SPEA) para comprobar su efectividad en el diseño de redes de distribución de agua potable, caso sector Viñani de la ciudad de Tacna, comparándolos con los resultados obtenidos con el Micro Algoritmo Genético Multiobjetivo (MAGMO). Mediante este proceso de optimización se pretende la búsqueda de la red de mínimo costo y máxima confiabilidad, que a su vez está sujeta a múltiples restricciones del tipo hidráulicas y normativas. Se trata de una red que tiene 01 reservorio, 20 circuitos básicos, 91 nodos y 139 tuberías. Los resultados indicaron que la red optimizada con MAGMO presentó un mejor desempeño para el diseño óptimo de redes de agua que la red optimizada con SPEA.
\end{abstract}

Palabras clave: Algoritmos genéticos, optimización multiobjetivo, redes de agua.

\begin{abstract}
The optimal design of distribution networks is the result of a detailed hydraulic study; which has been adapted to engineering criteria, economic requirements and current regulations. Yates, Templeman, \& Boffey (1984) affirm that it is necessary to find an approximate effective method that guarantees quality in the designs to extend the coverage of the potable water service. In this context, the Multiobjective Evolutionary Algorithm (SPEA) is applied to verify its effectiveness in the design of drinking water distribution networks, in the case Viñani sector of the city of Tacna, comparing them with the results obtained with the Multi-Objective Genetic Micro Algorithm (MAGMO). Through this optimization process, the search for the minimum cost and maximum reliability network is sought, which in turn is subject to multiple restrictions of the hydraulic and regulatory type. It is a network that has 1 reservoir, 20 basic circuits, 91 nodes and 139 pipelines. The results indicated that the network optimized with MAGMO presented a better performance for the optimal design of water networks than the network optimized with SPEA.
\end{abstract}

Keywords: Genetic algorithms, multiobjective optimization, water networks.

\footnotetext{
${ }^{1}$ Universidad Nacional Jorge Basadre Grohmann. Tacna-Perú. E-mail: epino68@hotmail.com

${ }^{2}$ Universidad Privada de Tacna. Tacna-Perú. E-mail: zarelaq@gmail.com

${ }^{3}$ Universidad Nacional Jorge Basadre Grohmann. Tacna-Perú. E-mail: lalfaror@unjbg.edu.pe

${ }^{4}$ Universidad Nacional Jorge Basadre Grohmann. Tacna-Perú. E-mail: neo_ceav@hotmail.com 


\section{INTRODUCCIÓN}

Reconociendo la importancia del agua como recurso natural limitado y teniendo en cuenta la perspectiva de crecimiento de las poblaciones urbanas, es necesario realizar un análisis integral que considere diversos factores que se hallan inmersos en esta problemática, tales como: costos, grado de confiabilidad y congruencia con la normativa para su posterior efectividad. Todo ello proporciona y asegura un diseño óptimo de redes de distribución de agua, rentabilidad, disponibilidad de tuberías, velocidad permisible, presión mínima, calidad del agua y distribución de la demanda.

En la actualidad, la aplicación de las técnicas heurísticas es escasa, así como las herramientas para determinar el modelo de optimización con mejor desempeño y que se pueda complementar con el diseño de redes de agua. Por un lado, existen ciertas restricciones para la elección de los diámetros como variables de decisión; y por otro, se está empleando un diseño que solo garantiza el suministro a los usuarios en condiciones normales, dejando la red vulnerable ante situaciones de emergencia.

Por lo tanto, el diseño óptimo de redes de distribución debe operar de manera eficiente en términos hidráulicos $\mathrm{y}$ económicos; esto se traduce en una mayor complejidad para dar solución con técnicas tradicionales. En tal sentido, la experiencia ingenieril se convierte en un valor agregado, ya que ofrece una amplia metodología de trabajo y posibilita la búsqueda de la optimización del diseño, que conduce a un mínimo costo en un tiempo computacional.

Es necesario señalar que para optimizar estos objetivos se han utilizado un sinfín de técnicas. En la última década se han popularizado las técnicas heurísticas, tal como se presenta en Sotelo \& Barán (2001) donde apuntan a minimizar cuatro objetivos: costo de la energía eléctrica consumida, costo de mantenimiento de las bombas, potencia máxima alcanzada y desnivel en el reservorio entre el inicio y el final del período de optimización siendo el SPEA el que brinda un conjunto de soluciones óptimas en vez de una solución y permite predecir las consecuencias en caso de realizar cambios en las características de las bombas, capacidad del tanque y demanda de consumo mediante simulaciones hechas con el algoritmo.

La optimización en un ambiente multicast pretende optimizar costo y retardo entre otras posibles variables, donde el SPEA tiene un mejor rendimiento para la resolución del problema de ingeniería de tráfico multicast (Talavera Solalinde, 2005). Por su parte, en un ambiente estático - como para un sistema de distribución de energía según García \& Mendoza (2011) y sistemas eléctricos de distribución según García et al. (2015) - el SPEA es una de las herramientas más relevantes por su flexibilidad y alta selectividad, lo que minimiza el problema y reduce los cálculos, así como el tiempo y la capacidad de cómputo requerida. De la misma manera, Potti \& Chitra (2011) manifiestan que el SPEA resulta superior; porque encuentra todas las soluciones posibles en una sola ejecución, ahorrando así tiempo de ejecución de la computadora. Además, agregan que el SPEA se aplica a redes de gran tamaño.

Otros trabajos se han enfocado en el estudio del Micro Algoritmo Genético usando optimización multiobjetivo, es decir, MAGMO. Según Peña (2002) facilita la solución de problemas con muy poca información, mejora la calidad de sus soluciones y aumenta la velocidad de ejecución.

Coello \& Toscano (2001) indican que el MAGMO junto con un archivo externo y un enfoque de diversidad basado en la distribución geográfica genera eficientemente los frentes de Pareto de varias funciones de prueba difíciles. Del mismo modo, Urzúa et al. (2014) sostienen que se maximiza la rentabilidad y se minimiza el riesgo, los resultados obtenidos señalan que es más eficiente que otros procesos de similares características como el NSGA II y PAES. Valle (2014) en su trabajo Optimización del diseño de redes de distribución de agua potable usando algoritmos genéticos con tres funciones objetivo. Caso: ViñaniTacna, sostiene que se logró evaluar y comparar la red de agua potable existente sin criterios de optimización con la resultante de la aplicación del método de Micro Algoritmos Genéticos con dos funciones objetivo de optimización: minimización de costo y maximización de la confiabilidad. De esta manera se obtuvo un costo de S/ 1933745 061; el cual si bien es mayor que el costo sin optimización, es decir, S/ 1311550083 , posee un índice de confiabilidad resiliente optimizado de 0,391 , valor menor que el obtenido sin optimización de 0,70 .

El empleo de algoritmos genéticos en la solución de las redes de distribución de agua ha permitido obtener un conjunto de soluciones, donde el diseñador finalmente deberá decidir por cuál optar en un grupo de redes de distribución de agua basado en dos funciones objetivos: minimización del costo y maximización de la confiabilidad, para la red de Hanoi, se obtuvo una confiabilidad de 0,497 y un menor costo que solución obtenida por Liong y Atiquzzam y Lenhsnet (Valle \& Pino, 2017).

En la optimización multiobjetivo de redes de distribución de agua no se ha abordado el estudio del desempeño del SPEA, por lo tanto, se propone la implementación de un algoritmo evolutivo multiobjetivo de frente de Pareto (SPEA) propuesto por Zitzler \& Thiele (1999), que sigue los principios de selección natural para generar una población inicial y una población externa. De este modo, ambas son evaluadas con el propósito de marcar las soluciones no dominadas de redes posibles; así como calcular el fitness de los individuos y aplicar posteriormente los operadores genéticos de selección por torneo y cruza para obtener una óptima generación. Asimismo, se presenta el Micro Algoritmo Genético Multiobjetivo (MAGMO) propuesto por Coello \& Toscano (2001), donde se posee un grupo de soluciones iniciales (denominados pobladores); a quienes se les aplican operadores como son los de selección, cruza, mutación y elitismo, a fin de obtener una nueva población evolucionada de la cual han sobrevivido los mejores pobladores y se ha descartado el resto. 
Estos métodos se desarrollan con el fin de encontrar un conjunto óptimo de soluciones que satisfaga dos objetivos: mayor confiabilidad y mínimo costo evaluando la configuración de la red con el método del gradiente y para el cálculo de la pérdida de carga por fricción, se empleó la ecuación de Darcy-Weisbach junto con la ecuación de Colebrook-White, resuelta con el método de Newton-Raphson.

El cálculo requerido para llegar al conjunto de soluciones se ha desarrollado con Mathlab R2012 con la finalidad de demostrar la efectividad del SPEA en la red del Sector Viñani de 01 reservorio, 20 circuitos básicos, 91 nodos y 139 tuberías.

\section{FUNCIONES OBJETIVO}

\section{Función de Confiabilidad}

La confiabilidad, según Todini (2000), es "la capacidad intrínseca que tiene una red de distribución de agua para superar las fallas repentinas y se mide como la proporción entre el excedente de potencia, que es entregado a los usuarios, y la máxima potencia que puede ser disipada en la red cuando se cumplen exactamente los criterios y condiciones de diseño. El índice de resiliencia es la capacidad de un sistema de reaccionar y superar un estado de esfuerzos o el incremento de la redundancia energética y decrecimiento de la energía disipada internamente en una red"'(p. 117).

El índice de resiliencia de una red de agua (Ir) está basado en la noción de que incrementarán las pérdidas de energía cuando la demanda también incremente, del mismo modo, se genere un aumento de rugosidad cuando ocurran fallas en las tuberías. Esto último se representa como la potencia adicional en cada nodo, de modo que exista un superávit suficiente de potencia que permita una red de distribución de agua más confiable; aquella no afectará el servicio de los usuarios durante su operación. El índice de resiliencia hace referencia a qué tanto excedente de energía hay en la red que está dado por la relación entre el superávit de potencia por unidad de peso proporcionado a la red y la máxima potencia por unidad de peso que podría ser disipada internamente por la red sin dejar de satisfacer la presión mínima. Finalmente se tiene la siguiente expresión:

$I_{R}=\frac{\sum_{j=1}^{n u} C_{j} Q_{j}\left(H_{j}-H_{j}^{*}\right)}{\sum_{i=1}^{n e} Q_{e} H_{e}-\sum_{j=1}^{n u} Q_{j} H_{j}^{*}}$

Siendo:

Ir : Índice de Resiliencia

$C j$ : Coeficiente de conectividad

$Q j$ : Caudal demandado en cada nudo

$Q e$ : Caudal entregado por el reservorio

$\mathrm{He}$ :Altura piezométrica del reservorio

$H j$ :Altura piezométrica real

\section{$H j^{*}$ : Altura piezométrica ideal}

un :Número de nudos

\section{ne : Número de reservorios}

Para poder evaluar la efectividad del algoritmo propuesto se ha invertido la representación del coeficiente de confiabilidad resiliente de la red al haberla transformado como una función de minimización, siendo el 0 el valor más alto mediante la siguiente expresión:

$$
I_{R^{\prime}}=1-I_{R}
$$

Teniendo en cuenta la variabilidad de diámetros, los cuales están en el rango de $90 \mathrm{~mm}$ y $400 \mathrm{~mm}$, se considerará un coeficiente de conectividad de 0, 6 .

\section{Función de Costo}

El costo de una red de distribución está determinado por el valor comercial de las tuberías y accesorios, que varía exponencialmente con el diámetro de la tubería más su costo de instalación (Mendoza \& Saldarriaga, 2010). Pudiendo expresarse una relación potencial respecto al diámetro de la siguiente manera:

$C_{T}=\sum_{i=1}^{N T} K_{1} L_{i} D_{i}^{K_{2}}$

Donde NT es el número de tuberías que confirman la red de distribución de agua; $L_{i}$ y $D_{i}$ son la longitud y el diámetro del iésimo tubo de la red; $\boldsymbol{k}$ y $\boldsymbol{x}$ son parámetros que se determinan por regresión, y para ello se debe considerar el costo del material en cuestión.

\section{OPTIMIZACIÓN MULTIOBJETIVO}

\section{Dominancia de Pareto}

Zitzler et al. (2000) afirman que "Los problemas reales usualmente requieren de la búsqueda de soluciones que satisfagan en forma simultánea múltiples criterios de desempeño u objetivos, los cuales pueden ser contradictorios. En este caso, para obtener la solución del problema basta con encontrar el mínimo o el máximo de una única función que resume todos los objetivos que se desean optimizar". (p. 180)

En los algoritmos de optimización multiobjetivo son comparadas dos soluciones a fin de establecer qué solución domina o no a la otra. Una alternativa de solución se considera mejor que otra si produce una solución objetivo de menor valor para el caso de minimización, o de mayor valor para el caso de maximización, y para los problemas multiobjetivo se considera al mismo tiempo funciones de minimizar y maximizar.

Por tanto, los algoritmos multiobjetivo introducen el operador de dominancia, el cual define que una solución $x_{1}$ domina otra solución $x_{1}$ si se cumplen las siguientes condiciones (Peñuela \& Granada, 2017): La solución $\boldsymbol{x}_{l}$ no es de menor calidad que $\boldsymbol{x}_{1}$ en todos los objetivos y la solución $x_{1}$ es estrictamente mejor que $\mathrm{x}$ en al menos uno de los objetivos. 


\section{Optimalidad de Pareto}

En el caso de múltiples objetivos, una solución que optimice un objetivo no optimizará los otros restantes. Esto lleva a un nuevo concepto denominado punto eficiente, también conocido como punto inferior o no dominado. Además, en la optimización con objetivos múltiples no existe una solución óptima única, sino un conjunto de soluciones no dominadas, para quienes no existen soluciones mejores en todos los objetivos del problema (Pareto, 1986). Es así que aplicando reiterativamente las reglas de dominancia sobre un conjunto cualquiera de soluciones de un problema de optimización multiobjetivo, se puede establecer cuáles son las alternativas dominantes, conocidas como conjunto no dominado. Las soluciones restantes forman parte del conjunto de soluciones dominadas. Asimismo, si se logra establecer cuál es el conjunto de soluciones dominantes a través de todo el espacio objetivo, entonces se habla de frente óptimo de Pareto (Peñuela \& Granada, 2007).

\section{Algoritmo Evolutivo Multiobjetivo de Frente de Pareto (SPEA)}

Se trata de un algoritmo que presenta características exclusivas frente a otros, pues guarda las soluciones no dominadas en una población externa, constituyendo así el frente de Pareto (Zitzler \& Thiele, 1999). Además, usa el concepto de Dominancia Pareto para asignar un valor fitness a los individuos, donde se incluye un conjunto de características en un único algoritmo que antes se habían presentado aisladas.

Es importante indicar que el fitness de un individuo está determinado a partir de las soluciones que se encuentran solo en la población de individuos no dominados y todas las soluciones que forman parte del conjunto externo de soluciones no dominadas que participan en la selección.

De igual manera, una población es un conjunto de individuos que poseen una configuración de diámetros para cada tramo en las tuberías de la red de distribución de agua. Por lo tanto, el SPEA ha sido aplicado a las redes de distribución de agua, y se ha establecido según su funcionamiento en los siguientes aspectos mostrados en la figura 1:

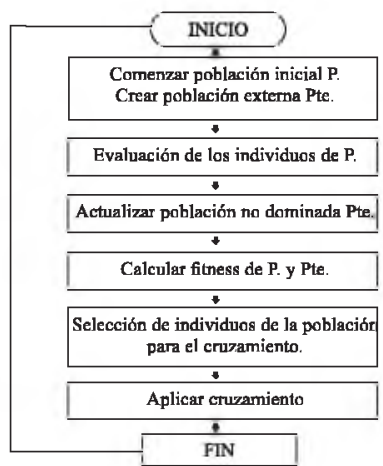

Figura 1. Diagrama de flujo del SPEA propuesto, adaptado de Zitzler \& Thiele(1999).

Fuente: Elaboración propia.

\section{FASES DEL ALGORITMO EVOLUTIVO (SPEA)}

\section{Generar población inicial (P) y crear población externa $\left(\mathbf{P t}_{e}\right)$}

El proceso empieza con la generación de una serie de individuos, se procede a realizar 2 etapas: Etapa 1: La población inicial $(\mathrm{P})$ es totalmente aleatoria, se genera un conjunto aleatorio de soluciones, se escogen cadenas al azar, pudiendo contener cada gen uno de los posibles diámetros comerciales para la red propuesta. Etapa 2: Simultáneamente con la carga de la población inicial (P) se crea la población externa $\left(\mathrm{Pt}_{e}\right)$ que albergará el frente de Pareto hallado dentro del proceso de optimización como un contador para retener las soluciones no dominadas previamente encontradas; en cada generación se copia en este archivo las soluciones no dominadas y se borran las que pasan a ser dominadas.

\section{Evaluar individuos de $\mathbf{P}$}

Los individuos son ordenados en base a su aptitud. Esta evaluación de una solución significa calcular el valor de las funciones objetivo y la violación de restricciones. Estos se reproducen atendiendo a una serie de criterios que otorgan mayor probabilidad de reproducirse a los mejores individuos y menor probabilidad de reproducirse a los de menos calidad.

\section{Actualizar población no dominada $\left(\mathbf{P t}_{\boldsymbol{e}}\right)$}

Todos los individuos no-dominados de la población se copian en el archivo, los individuos dominados o duplicados se eliminan del archivo durante esta actualización. Entonces, una vez definidos los parámetros del algoritmo, población inicial y población externa, se evalúa el dominio de los individuos que componen la población general, agregando los individuos no dominados a la población externa; la cual contendrá el frente de Pareto resultante del proceso de optimización.

Cálculo de adaptabilidad de los individuos $\mathbf{P y} \mathbf{P t}_{e}$ La asignación de la adaptabilidad es un proceso de 2 etapas:

Etapa 1: La población externa es evaluada de acuerdo a las funciones objetivo, donde a cada solución $i \in P t$ se le asigna un valor real; $S_{i} \in(0,1)$ será llamado strength; por último, es proporcional al número $n_{\mathrm{i}}$.

$$
S_{i}=\frac{\mathrm{n}_{\mathrm{i}}}{(\mathrm{N}+1)}
$$

$\mathrm{n}_{i}$ : Número de soluciones dominadas por $i$ en la población $P$

$\mathbf{N}$ : Número de individuos

Etapa 2: A cada individuo $\mathrm{j}$ perteneciente a la población externa se le calcula su aptitud, dependiendo de los valores de fuerza asignados a los individuos del archivo externo que lo dominan. Se suma 1 a ese total para asegurar que los miembros de Pt tengan mejor strength que los miembros de población inicial. 


$$
\mathrm{f}_{\mathrm{j}}=1+\sum_{i, i>j} S_{i}
$$

Una parte esencial del algoritmo es la asignación de aptitud que se divide en dos partes, dependiendo de la población que se está asignando. Cuanto mayor sea el fitness de una solución, mayor será la probabilidad de que la misma sea seleccionada (García \& Mendoza, 2011). A los individuos de la población se les asigna un valor de fitness por cada solución del archivo externo y su fuerza S . El proceso se realiza de modo que los individuos dominantes y más representativos tengan mejores valores de fitness. De esta manera ambas poblaciones $(P$ y $P t)$ se ejecutan para aplicar los operadores genéticos de selección y cruzamiento, manteniendose constante en tamaño de la población $P$.

\section{Selección por torneos del conjunto $P$}

La población y el conjunto externo se combinan basados en su valor de aptitud strength, donde el mejor será seleccionado, es decir, los cromosomas con mejor aptitud a la función objetivo y restricciones impuestas tienen mayor probabilidad de ser seleccionados. Una vez que se han determinado los individuos, se procede a la selección de los padres mediante la metodología de torneo - en la cual los pobladores se dividen en dos pares $y$, cada par es sometido a contienda- Los vencedores de cada torneo son los únicos facultados para obtener descendencia, en otras palabras, el cruzamiento.

\section{Aplicar cruzamiento \\ Los ocho padres resultantes anteriormente son sometidos al operador genético de cruzamiento para la obtención de cuatro hijos. El proceso para la obtención de un hijo consiste en la combinación de sus genes (diámetros en cada tramo de tuberia). El método que ha sido empleado es la combinación de un punto aleatorio, donde para cada tramo se obtiene un número al azar que determina si se heredará el diámetro del Padre $\mathrm{N}^{\circ} 01$ o el Padre $\mathrm{N}^{\circ}$ 02. El proceso del operador genético se repite para la obtención de los cuatro hijos.}

\section{DISEÑO ÓPTIMO DE REDES DEAGUA}

\section{Restricciones Hidráulicas}

Según Villalba \& Saldarriaga (2005), se tiene las siguientes leyes y restricciones hidráulicas: 1) Ley de Conservación de Masa en cada nodo, la sumatoria de flujos que entran y salen de un nodo es igual a cero. (Aportes, Demandas y Caudales). 2) Ley de Conservación de Energia en cada circuito, la sumatoria de las pérdidas de energía por fricción a lo largo de los tramos que pertenecen a una malla debe ser igual a cero.

\section{Restricciones Técnico-Normativas}

La normatividad peruana para el disex́o de redes de distribución de agua para consumo humano está dada por el Reglamento Nacional de Edificaciones en su norma OS.050.

\section{Presión en los nudos}

Según la normatividad peruana, la presión mínima y máxima en cualquier nudo que conforme una red de distribución de agua potable deben estar entre 10 y 50 mca respectivamente.

\section{Velocidad en los tramos}

Las velocidades máximas son de $3,00 \mathrm{~m} / \mathrm{s}$; y en casos justificados de $5,00 \mathrm{~m} / \mathrm{s}$ para evitar la erosión excesiva.

\section{Restricciones comerciales}

Villalba \& Saldarriaga (2005) señalan que los diámetros solo pueden tomar valores discretos dados por la disponibilidad de diámetros de cada fabricante de tuberías.

\section{Restricciones adicionales}

El método idóneo debe ser capaz de encontrar una solución apropiada para una amplia variedad de topologías, demandas en los nodos, topografia, entre otros (Villalba \& Saldarriaga, 2005). Asimismo, debe ser capaz de encontrar una "correcta" solución en un tiempo "razonable" según el tamaño de la red.

\section{Metodología Propuesta}

Para la comprobación de la metodología adoptada, se ha planteado su aplicación en la red de distribución de agua potable del Sector Vinani en la ciudad de Tacna, figura 2. Esta red permitió realizar la comparación de resultados entre el MAGMO y el SPEA.

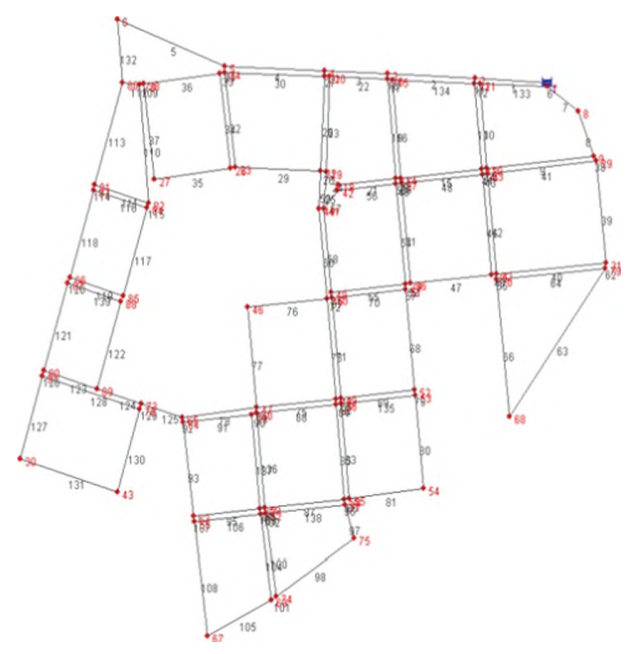

Figura 2. Red de Sector Viñani. Valle, A. (2014). Optimización del Diseño de Redes de Distribución de agua potable, usando algoritmos genéticos con tres funciones objetivo. Caso: Viñani, Tacna, Perí.

Fuente: Valle,A.(2014).

Esta red tiene 01 reservorio, 20 circuitos, 91 nodos y 139 tuberías; se ensayaron 08 diámetros comerciales disponibles, el número de soluciones posibles es de $3,38 \times 10$. Para la aplicación de estos algoritmos se empleó el método de cálculo hidráulico del Gradiente, en forma conjunta con las ecuaciones de DarcyWeisbach y Colebrook-White. El valor de rugosidad absoluta considerado es de $1,5 \times 10 \mathrm{~m}$ y viscosidad del fluido de $1,410 \mathrm{~m} 7 \mathrm{~s}$ y se tomará como presión mínima requerida en los nodos por la normativa peruana de 10 mca. 
Los parámetros para la optimización del SPEA introducidos fueron los siguientes:

- Número de generaciones

- Número de genes de los pobladores

- Tamaño de individuos

- Número de torneos binarios

$: 4$

- Cruzamiento

: 1 Punto

- Números de hijos en 0,28 , pero con un costo mayor en $\mathrm{S} / 1219253,03$. La tabla 1 muestra que las menores velocidades se dan con la solución de la red sin optimización $2,59 \mathrm{~m} / \mathrm{s}$.

Las presiones resultantes en los nodos de la red del sector Viñani muestran que la presión mínima para la red aplicando el SPEA se da en el nodo $\mathrm{N}^{\circ} 19$ con un valor de 12,08 mca y una presión máxima en el nodo $\mathrm{N}^{\circ} 66$ con $68,14 \mathrm{mca}$. En la solución de la red aplicando MAGMO es en el nodo $\mathrm{N}^{\circ} 32$ con un valor de 23,275 mca y una presión máxima en el nodo $\mathrm{N}^{\circ} 21$ con 68.46 mca; en la solución de la red sin optimización en el nodo $\mathrm{N}^{\circ} 14$ con un valor de 10,14 mca y una presión máxima en el nodo $\mathrm{N}^{\circ} 66$ de 45,49 mca. La solución con MAGMO ha tenido un valor de presión mínima y máxima por encima de las otras dos soluciones.

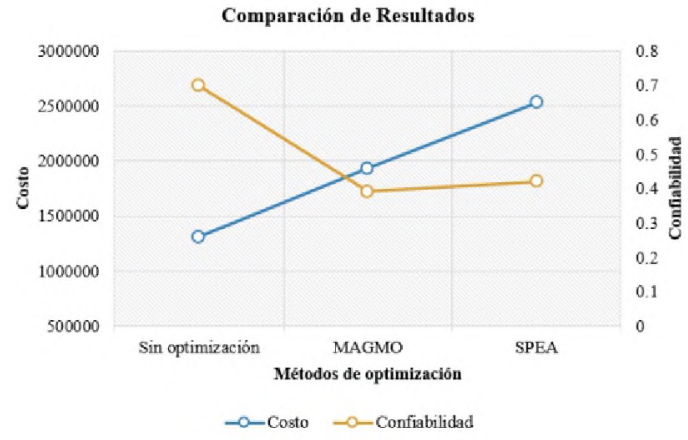

Figura 4. Comparación de resultados según métodos utilizados.

Fuente: Elaboración propia.

Según la figura 4, la solución MAGMO y SPEA representan un mejor valor para la confiabilidad de las redes de distribución de agua a comparación de la red sin optimización. Entonces, podemos decir que la elección del grupo de redes resultantes finales siempre será una red optimizada al estar ubicadas todas las respuestas en el frente de Pareto. Por tanto, puede elegirse la que tenga el menor costo posible con la contrapropuesta de una baja confiabilidad; la de mayor confiabilidad, que generaría un alto costo para su implementación; o elegir una red con características intermedias. Para la zona de estudio se pretende dar un servicio eficiente y confiable en el sector Viñani sin despreciar un costo apropiado.

\section{CONCLUSIONES}

El Algoritmo Evolutivo Multiobjetivo de Frente de Pareto (SPEA) para el diseño óptimo de redes de distribución de agua es adecuado ya que la elección de los diámetros cumple con las restricciones hidráulicas, las restricciones comerciales de disponibilidad de diámetros, la fiabilidad de la red poco vulnerable a todo tipo de fallas que reducen la eficiencia de las redes y generan una pérdida económica a los organismos operadores del sistema de distribución de agua. El SPEA se aplica a redes de gran tamaño, minimiza el problema y reduce los cálculos, así como el tiempo. 
Realiza un análisis integral que implica una optimización simultánea de dos funciones objetivo: Máxima confiabilidad y mínimo costo para un diseño óptimo que asegura la calidad del método. De este modo, se puede adoptar cualquier alternativa de acuerdo a las necesidades.

Se evidencia notables mejoras respecto a los métodos tradicionales de diseño que se limitan a verificar condiciones hidráulicas y no pretenden optimizar el diseño de la red de agua potable. El costo estimado de la red optimizada es económicamente superior a la red sin optimización (S/ 2530 803,86 vs S/ 1311 550,83); sin embargo al incluir como parámetro a optimizar en el diseño la confiabilidad de la red de agua potable, se hizo evidente que la red más confiable es la optimizada con un índice de resiliencia de 0,42 contra un índice de resiliencia de 0,70 vulnerable a todo tipo de fallas.

E1 Micro Algoritmo Genético Multiobjetivo (MAGMO) tiene mejor desempeño que el Algoritmo Evolutivo Multiobjetivo de Frente de Pareto (SPEA), los resultados indicaron que con el algoritmo SPEA tiene una confiabilidad resiliente de 0,42 y un costo estimado de S/ 2530803,86 el cual es superior al costo estimado con la implementación del MAGMO en $\mathrm{S} / 597058,25$, además una confiabilidad de 0,391 con menos probabilidad de fallas.

Ambos algoritmos tienen capacidades similares para encontrar soluciones cercanas al conjunto de soluciones Pareto óptimas, pero se evidencia que el SPEA no es efectivo para el diseño óptimo de redes; ello puede resultar debido a: (a) La forma de asignación del strength y la asignación posterior de la aptitud puesto que, cuando existe un único elemento dominante en la población externa, el algoritmo es casi una búsqueda ciega; cuando existen demasiados elementos dominantes, ya no se realiza la búsqueda eficientemente y se pierde la posibilidad de ir encontrando siempre mejores soluciones. (b) Existe una convergencia prematura que no mantiene la diversidad en la población para lograr así un conjunto no dominado bien distribuido y amplio.

El método del gradiente en la actualidad es el mejor método de cálculo de redes debido a que la manipulación de las matrices dispersas reduce el tiempo de cálculo en el computador, lo que no es posible con otros métodos; además, toma en cuenta para el diseño de tuberías las pérdidas de energía y conservación de la masa.

\section{REFERENCIAS BIBLIOGRÁFICAS}

Coello, C. \& Toscano, G. (2001). Multiobjective optimization using a micro-genetic algorithm. Proceedings of the genetic and evolutionary computation conference, 274-282.

García, C. A. \& Mendoza, S. (2011). Implementación de un Algoritmo Evolutivo en la Planeación de Inversiones de un Sistema de Distribución de Energía. Scielo.

García, C. A., García, E., \& Villada, F. (2015). Implementación del Algoritmo Evolutivo
Multi-Objetivo de Frente de Pareto (SPEA) para la Planeación de Sistemas Eléctricos de Distribución incluyendo Huecos de Voltaje. Información Tecnológica, 26(5), 155-168.

Mendoza, F. \& Saldarriaga, J. G. (2010). Diseño optimizado de redes de distribución de agua potable incluyendo análisis de costo mínimo versus resiliencia en la red. XXIV Congreso Latinoamericano de Hidráulica. Punta del Este, Uruguay.

Pareto, V. (1986). Cours DEconomie Politique. I,II. Rouge,Lausanne.

Peña, J. J. (2002). Desarrollo de un Applet del Micro Algoritmo Genético Usando Optimización Multiobjetivo (Tesis de Pregrado). Mérida, Universidad Autónoma de Yucatán, Yucatán.

Peñuela, C. \& Granada, M. (2007). Optimización multiobjetivo usando un algoritmo genético y un operador elitista basado en un ordenamiento no-dominado (NSGAII). Scientia et Technica Año XIII(35).

Potti, S. \& Chitra, C. (2011). Strength Pareto Evolutionary Algorithm based MultiObjective Optimization for Shortest Path Routing Problem in Computer Networks. Journal of Computer Science, 7(1), 17-26.

Sotelo, A. \& Barán, B. (2001). Optimización de los costos de bombeo en sistemas de suministro de agua mediante un algoritmo evolutivo multiobjetivo combinado. Universidad Nacional deAsunción.

Talavera Solalinde, F. (2005). Comparación de algoritmos evolutivo multi-objetivos en un ambiente multicast (Tesis de Pregrado). Universidad Católica Nuestra Señora de la Asunción, Paraguay.

Todini, E. (2000). Looped water distribution networks design using a resilience index based heuristic approach. Urban Water.

Urzúa, M. G., Melillanca, E. T., Gálvez, P. G. \& Caamaño, G. P. (2014). Optimización de portafolios accionarios a través de un micro algoritmo genético. Industrial Data, 10(2), 012-020.

Valle Castro, A. P. (2014). Optimización del Diseño de Redes de Distribución de agua potable, usando algoritmos genéticos con tres funciones objetivo. Caso: Viñani-Tacna (Tesis de Pregrado). Tacna, Perú.

Valle, A. \& Pino, E. (Edits.). (2017). RIBAGUARevista Iberoamericana del Agua, 3(1), 118.

Villalba, G. \& Saldarriaga, J. (2005). Algoritmos de Optimización Combinatoria (AOC) aplicados al diseño de redes de distribución de agua potable. Revista de Ingeniería(22), 118-125.

Yates, D. F., Templeman, A. B. \& Boffey, T. B. (1984). The computational complexity of the 
problem of determining least capital cost designs for water supply networks. Engg. Optimization, 7(2), 142-155.

Zitzler, E. \& Thiele, L. (1999). Multiobjective Evolutionary Algorithms: A Comparative Case Study and the Strength Pareto Approach. IEEE Transactions on Evolutionary Computation, 3(4).

Zitzler, E., Deb, K. \& Thiele, L. (2000). “Comparison of Multiobjective Evolutionary Algorithms:Empirical Results. Evolutionary Computation, 8, 173-195. 